T. Tillin • N. Forouhi • D. G. Johnston •

P. M. McKeigue • N. Chaturvedi • I. F. Godsland

\title{
Metabolic syndrome and coronary heart disease in South Asians, African-Caribbeans and white Europeans: a UK population-based cross-sectional study
}

Received: 3 August 2004 / Accepted: 7 November 2004 / Published online: 10 March 2005

C) Springer-Verlag 2005

\begin{abstract}
Aims/hypothesis: The aim of this study was to study differences in the prevalence of the metabolic syndrome and its associations with prevalent CHD according to ethnicity and sex. Methods: We performed a combined analysis of two population-based cross-sectional studies conducted between 1988 and 1991 that followed identical protocols. Participants (aged 40-69 years) comprised 2,346 Europeans ( $76 \%$ male), 1,711 South Asians ( $83 \%$ male) and 803 African-Caribbeans (57\% male) resident in west London. Fasting blood, overnight urine collection, clinical and anthropometric measurements were performed. Clinical history or major ECG changes defined prevalent CHD. The metabolic syndrome was defined according to the criteria recommended by the World Health Organization (WHO) and the National Cholesterol Education Programme (NCEP). Results: The prevalence of the metabolic syndrome was highest in South Asians (WHO, men 46\%, women 31\%; NCEP, men 29\%, women 32\%) and lowest in European women (WHO, 9\%; NCEP, 14\%). The prevalence of CHD
\end{abstract}

Electronic Supplementary Material Supplementary material is available in the online version of this article at http://dx.doi. org/10.1007/s00125-005-1689-3

T. Tillin $(\bowtie) \cdot$ N. Chaturvedi

National Heart and Lung Institute,

Imperial College St Mary's Campus,

59-61 North Wharf Road,

London, W2 1LA, UK

e-mail: t.tillin@imperial.ac.uk

Tel.: +44-20-75943396

Fax: +44-20-75943392

N. Forouhi

Medical Research Council Epidemiology Unit,

Strangeways Research Laboratory,

Cambridge, UK

D. G. Johnston · I. F. Godsland

Endocrinology and Metabolic Medicine Section,

Imperial College St Mary's Campus,

London, UK

P. M. McKeigue

Conway Institute, University College,

Dublin, Ireland was $10 \%$ in South Asian men, 9\% in European men, 5-6\% in African-Caribbeans and European women, and 2\% in South Asian women. The metabolic syndrome was associated with prevalent CHD in European men [NCEP, odds ratio $(\mathrm{OR})=1.6,95 \%$ CI $1.2-2.4 ; \mathrm{WHO}, \mathrm{OR}=1.7$, 95\% CI 1.2-2.5] and South Asian men (NCEP, OR=2.1, 95\% CI 1.5-3.1; WHO, OR=1.6, 95\% CI 1.1-2.3). Associations with CHD were weaker in African-Caribbeans and were inconsistent among European women. Conclusions/ interpretation: The current definitions of the metabolic syndrome give an inconsistent picture of cardiovascular disease risk when applied to different ethnic groups within the UK. Prospective studies are needed to validate workable ethnic-specific definitions.

Keywords Coronary heart disease $\cdot$ Epidemiological studies $\cdot$ Ethnic groups $\cdot$ Metabolic syndrome $\cdot$ Prevalence

Abbreviations ATPIII: Adult Treatment Panel III - CHD: Coronary heart disease - CVD: Cardiovascular disease HOMA: Homeostasis model assessment - LBBB: Left bundle branch block - NCEP: National Cholesterol Education Programme - OR: Odds ratio - WHO: World Health Organization

\section{Introduction}

In 2002, CHD and cerebrovascular disease together accounted for $30 \%$ of all deaths in England and Wales [1]. However, there are striking differences in the risks of CHD and cerebrovascular disease between ethnic groups. In England and Wales, compared with the general population, mortality from CHD is $50 \%$ higher in South Asians and is $50 \%$ lower in African-Caribbeans. Mortality from cerebrovascular disease is highest in African-Caribbeans and higher in South Asians when compared with Europeans [2].

During the late 1980s, the concept emerged of a syndrome of metabolic disturbances centred on insulin resistance and including impaired glucose homeostasis, central adiposity, dyslipidaemia and hypertension [3]. We have 
previously reported higher prevalences of some individual components of the metabolic syndrome in UK South Asians and African-Caribbeans compared with Europeans [4]. However, two composite definitions of the metabolic syndrome have since been proposed by the National Cholesterol Education Programme (NCEP) Adult Treatment Panel III (ATPIII) report [5] and by the World Health Organization (WHO) [6] (Table 1). These composite measures have been shown to predict CHD risk, largely in European populations [7-13].

The aim of the present study was to investigate the prevalence of the metabolic syndrome and its association with CHD in South Asian and African-Caribbean populations in the UK.

\section{Subjects and methods}

Study design We studied the prevalence of the metabolic syndrome and its association with CHD according to ethnicity and sex using data collected during two cross-sectional population-based studies that were conducted to identical protocols between 1988 and 1991 (the Brent and Southall studies). Both studies have previously been described in detail $[4,14]$ and were approved by local research ethics committees.

Subjects Participants were aged between 40 and 69 years and comprised 2,346 white Europeans (76\% male), 1,711 South Asians (83\% male) and 803 African-Caribbeans (57\% male). The term 'South Asian' describes people of Indian, Pakistani or Bangladeshi origin. Recruitment was mainly from random samples from GP practice lists stratified according to ethnicity and sex. Sixteen percent $(n=795)$ of participants were recruited from four factories in west London, which were chosen based on the ethnic mix of their workforce. The Southall study, the source of all South Asians in this study, preferentially sampled men to address the original specific study objectives and aimed to recruit equal numbers of European and South Asian men; AfricanCaribbeans who appeared in the sampling frame were also included. A target random sample (ethnicity stratified) of 600 women supplemented the original sample. The Brent study aimed to recruit equal numbers of European and African-Caribbean men and women.

Assessments and biochemical measurements Ethnic group was assigned by the interviewer on the basis of name, appearance and country of birth, supplemented with direct enquiry in cases of doubt. Participants attended a local hospital or factory medical centre between 07:00 and 11:00 h following an overnight fast. Height, weight and blood pressure were measured, a resting ECG performed, and blood samples taken for measurement of plasma triglycerides, cholesterol, HDL cholesterol, glucose and insulin. ECGs were coded by two experienced coders who were blinded to all other information on each subject. Timed overnight urine collections were carried out and urine albumin measured. For participants not known to be diabetic, an oral glucose load was given and plasma glucose, insulin and triglycerides were measured $2 \mathrm{~h}$ later. Measurements for the two studies were made at the same hospital chemical pathology laboratory. A self-completion questionnaire included items on socioeconomic status, medical history and lifestyle.

Analyses Self-reported diabetes was taken to indicate known diabetes. Newly diagnosed type 2 diabetes, IGT or IFG were ascertained using both fasting and post-load values according the criteria defined by the WHO in 1999 [6].

Insulin resistance was assessed in the fasting state in terms of the fasting plasma insulin concentration [15] and the homeostasis model assessment (HOMA) index of insulin resistance, which is calculated using the following equation: fasting plasma glucose $(\mathrm{mmol} / \mathrm{l}) \times$ fasting plasma insulin (pmol/1)/22.5 [16]. OGTT glucose and insulin responses were used to estimate insulin resistance. The glucose and insulin concentrations at $2 \mathrm{~h}$ acted as indices of the overall OGTT responses, and these values were used in the formula proposed by Matsuda and DeFronzo [17]: square root of ([fasting glucose $(\mathrm{mmol} / \mathrm{l}) \times$ fasting insulin $(\mathrm{pmol} / \mathrm{l})] \times[$ mean OGTT glucose $(\mathrm{mmol} / \mathrm{l}) \times$ mean OGTT insulin $(\mathrm{pmol} / \mathrm{l})] / 3,330)$. Matsuda's formula can only be applied to participants who underwent OGTT (i.e. those who were not known to be diabetic in this study). Both the HOMA and Matsuda indices include glucose concentration in their calculation, which diminishes confounding due to

Table 1 Metabolic syndrome definitions

\begin{tabular}{|c|c|c|}
\hline WHO definition [6] & & NCEP ATPIII definition [5] \\
\hline At least one of the following & Plus two or more of the following & At least three of the following \\
\hline Fasting plasma glucose $\geq 6.1 \mathrm{mmol} / 1$ & Blood pressure $\geq 140 \mathrm{mmHg} / 90 \mathrm{mmHg}$ & Fasting plasma glucose $\geq 6.1 \mathrm{mmol} / 1$ \\
\hline $\begin{array}{l}\text { Insulin resistance under euglycaemic--hyperinsulinaemic } \\
\text { clamp conditions in the upper quartile for the } \\
\text { background population under investigation }\end{array}$ & $\begin{array}{l}\text { Triglycerides } \geq 1.7 \mathrm{mmol} / 1 \text { and/or HDL } \\
\text { cholesterol }<0.9 \mathrm{mmol} / 1 \text { (men), } \\
<1.0 \mathrm{mmol} / 1 \text { (women) }\end{array}$ & Blood pressure $\geq 130 \mathrm{mmHg} / \geq 85 \mathrm{mmHg}$ \\
\hline IGT $(2-\mathrm{h}$ glucose $\geq 7.8 \mathrm{mmol} / \mathrm{l})$ & $\begin{array}{l}\text { WHR }>0.90 \text { (men), }>0.85 \text { (women) } \\
\text { and/or BMI }>30 \mathrm{~kg} / \mathrm{m}^{2}\end{array}$ & Triglycerides $\geq 1.7 \mathrm{mmol} / 1$ \\
\hline Known or newly diagnosed diabetes & $\mathrm{AER} \geq 20 \mu \mathrm{g} / \mathrm{min}$ & $\begin{array}{l}\text { HDL cholesterol }<1.04 \mathrm{mmol} / 1 \text { (men), } \\
<1.29 \mathrm{mmol} / 1 \text { (women) } \\
\text { Waist circumference }>102 \mathrm{~cm} \text { (men), } \\
>88 \mathrm{~cm} \text { (women) }\end{array}$ \\
\hline
\end{tabular}


deficient beta cell function at glucose levels below the conventional upper limits for normoglycaemia [18]. The HOMA estimates of insulin resistance are primarily influenced by hepatic insulin resistance [16], whereas the Matsuda index values, determined in the glucose-stimulated state, are primarily influenced by peripheral insulin resistance (mainly at the level of muscle). The use of these three estimates of insulin resistance (fasting hyperinsulinaemia, and HOMA and Matsuda indices) strengthens the ability of the study to detect significant insulin resistance. Unless otherwise stated, the results shown for the WHO metabolic syndrome include Matsuda's method of estimating insulin resistance [17] based on the entire non-diabetic study population.

Microalbuminuria was defined by a cutoff value for AER of $>20 \mu \mathrm{g} / \mathrm{min}$, as recommended by the WHO, rather than the albumin/creatinine ratio, which is prone to sex- and ethnic-specific variation due to its dependency on muscle mass and dietary protein intake [19].

Participants receiving treatment for hypertension were included in the hypertension categories for both definitions of metabolic syndrome, irrespective of their actual blood pressure measurements.

Age-standardised prevalences of the metabolic syndrome according to the two definitions were determined in the study population as a whole and in those without known or newly diagnosed diabetes in each of the groups classified according to ethnicity and sex.

CHD was defined as the presence of either a history of doctor-diagnosed angina or heart attack, or the presence of major Q-wave or left bundle branch block (LBBB) changes on ECG.

Logistic regression models were used to describe the associations between prevalent CHD and the metabolic syndrome. These associations are described in the study population as a whole and in those without known or newly diagnosed diabetes in each ethnic group, stratified according to sex and adjusted for age and smoking status (current, ex and never).

Age-adjusted averages were calculated for each component of the metabolic syndrome; natural log transformations were used to derive geometric means for variables with non-normal distributions. The average values for each component for participants with and without CHD, stratified according to ethnic group and sex, were compared. Statistical significance was accepted at less than 5\%.

\section{Results}

Prevalence of the metabolic syndrome and components The age-standardised prevalence of the metabolic syndrome was highest in South Asians (WHO, men 46\%, women $31 \%$; NCEP, men $29 \%$, women $32 \%$ ) and lowest in European women (WHO, 9\%; NCEP, 14\%) (Fig. 1a, b, Table 2). With regard to sex, the prevalence of the metabolic syndrome as defined by the WHO was three times higher in African-Caribbean women than in European women, but only 1.5 times higher in African-Caribbean men. This sex-
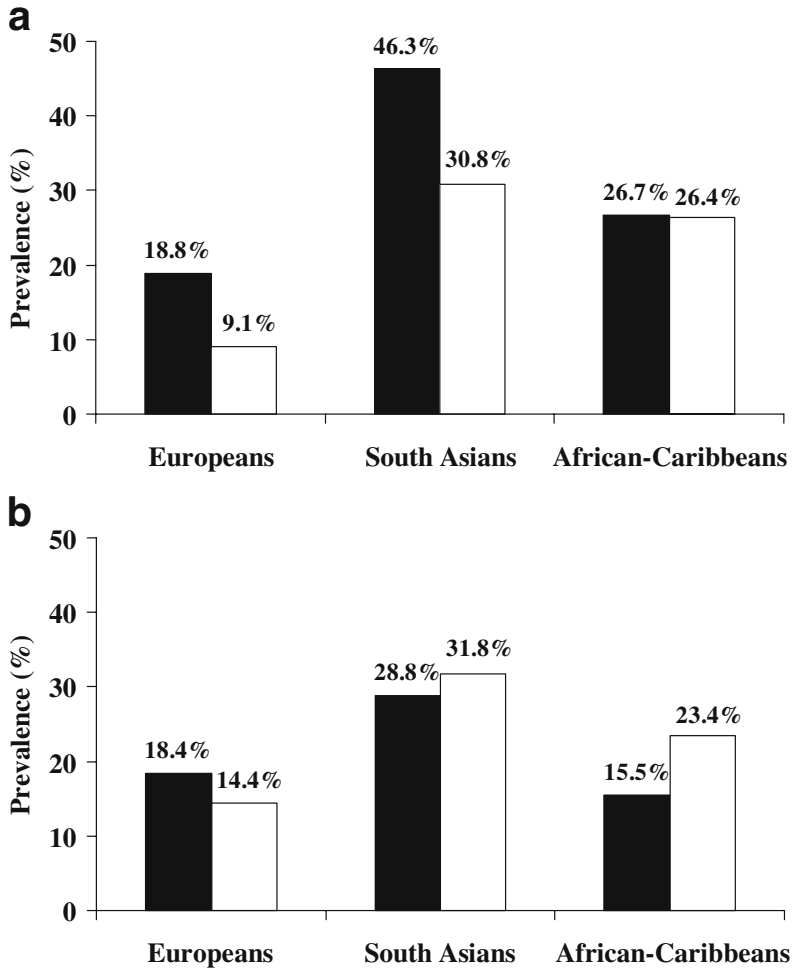

Fig. 1 Age-standardised prevalences of the metabolic syndrome as defined by the WHO criteria (a) or the NCEP criteria (b) in men (black bars) and women (white bars) divided according to ethnicity

ethnicity interaction was significant $(p<0.001)$. A similar interaction was obtained for the NCEP definition ( $p=$ 0.001 ). The prevalence of metabolic syndrome was lower in all groups when participants with diabetes were excluded, although the same patterns remained in terms of ethnic group and sex (Table 2).

While both definitions provided equivalent estimates for prevalence in European men, use of the NCEP definition provided a lower prevalence than the WHO definition in South Asian and African-Caribbean men. In contrast, the prevalence according to the NCEP definition was higher in European women and was similar for both definitions in South Asian and African-Caribbean women.

There was significant discordance between the two definitions of the metabolic syndrome. Of 1,540 participants identified by either definition, $767(50 \%)$ were identified by both the NCEP and WHO definitions, 498 (32\%) were identified by the WHO definition alone, and $275(18 \%)$ were identified by the NCEP definition alone $(p<0.001)$. The discordance between definitions was most marked in South Asian and African-Caribbean men.

Impaired glucose homeostasis was most prevalent in South Asians and African-Caribbeans (Table 2). The impaired glucose homeostasis category defined by the WHO identified 1,909 participants, whereas that defined by the NCEP identified only 1,070 participants; again, discordance was most marked in South Asians and AfricanCaribbeans. We compared the prevalences of the metabolic syndrome as defined by the WHO using either Matsuda's formula, the HOMA formula or fasting insulin concentra- 


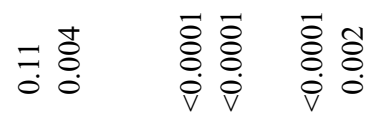

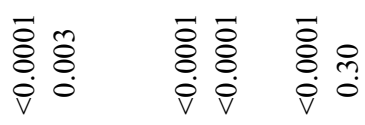

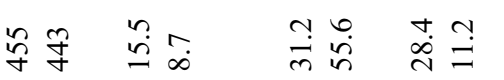

ิㅗํ

$\stackrel{2}{2}$

要

穵

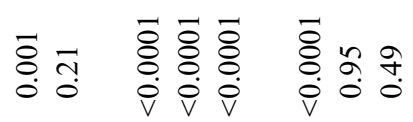

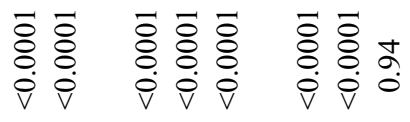

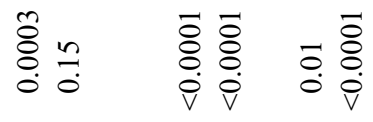

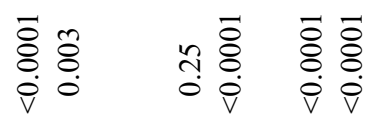

市

बे山

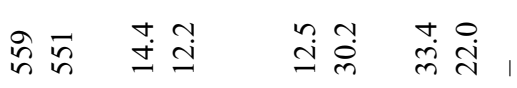

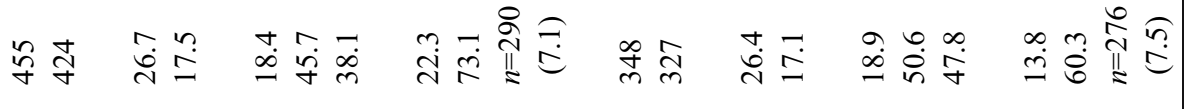

กิ

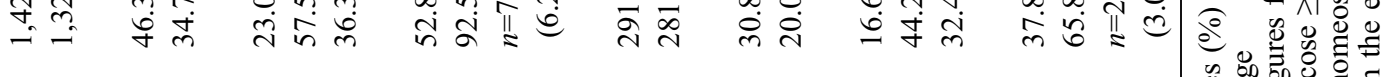

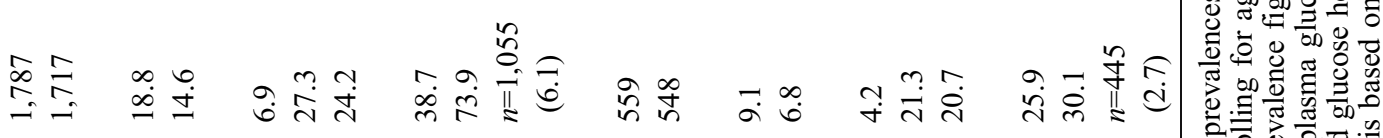
$=0$

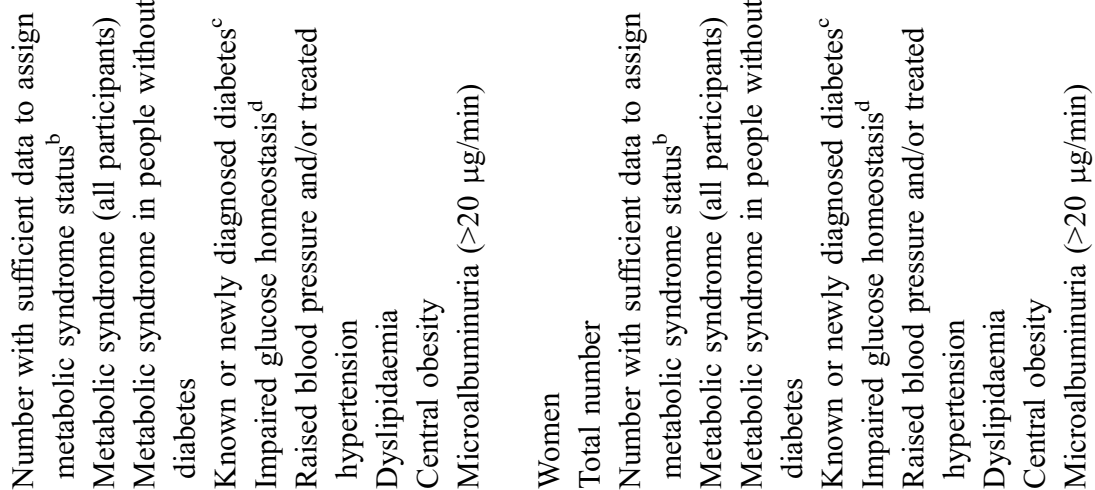

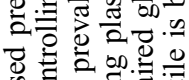
:

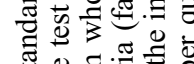

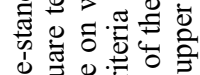

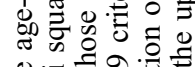
흥 응

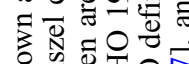

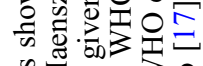
造

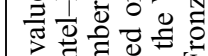

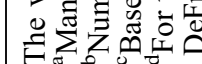


tions to estimate insulin resistance. There was significant discordance between these three values with respect to the identification of insulin-resistant individuals, but this had little effect on either the absolute prevalence of the metabolic syndrome or its associations with CHD within ethnic groups stratified according to sex (results not shown). The prevalence of dyslipidaemia according to ei- ther definition was significantly higher in South Asians and significantly lower in African-Caribbeans compared with that in Europeans. Of those with the NCEP definition of the metabolic syndrome, $28 \%$ of Europeans and South Asians and $9 \%$ of African-Caribbeans had sufficient dyslipidaemia such that only one other component was required to identify them as metabolic syndrome 'cases'. The WHO central

Table 3 Prevalence of smoking and CHD, and odds ratios for associations between WHO and NCEP ATPIII definitions of the metabolic syndrome and prevalent CHD

\begin{tabular}{|c|c|c|c|c|c|}
\hline & \multicolumn{2}{|l|}{ Europeans } & \multirow{2}{*}{$\frac{\text { South Asians }^{\mathrm{a}}}{\text { Men }}$} & \multicolumn{2}{|l|}{ African-Caribbeans } \\
\hline & Men & Women & & Men & Women \\
\hline \multicolumn{6}{|l|}{$\begin{array}{l}\text { Age-standardised } \\
\text { prevalence, } \%(95 \% \mathrm{CI})\end{array}$} \\
\hline $\begin{array}{l}\text { Major Q-waves and/or } \\
\text { LBBB on ECG }\end{array}$ & $4.1(3.2-4.9)$ & $1.8(0.6-2.8)$ & $4.8(3.9-6.2)$ & $2.2(0.8-3.6)$ & $1.4(0.2-2.7)$ \\
\hline $\begin{array}{l}\text { Previously diagnosed } \\
\text { (by doctor) CHD }\end{array}$ & $7.0(5.8-8.1)$ & $4.0(2.4-5.5)$ & $6.9(5.6-8.3)$ & $3.1(1.5-4.8)$ & $3.5(0.2-5.5)$ \\
\hline $\begin{array}{l}\text { CHD defined by major ECG } \\
\text { changes and/or previous } \\
\text { diagnosis }\end{array}$ & $9.1(7.8-10.4)$ & $5.6(3.7-7.4)$ & $9.8(8.2-11.4)$ & $5.0(2.9-7.0)$ & $4.9(2.6-7.3)$ \\
\hline Current smoking & $31.8(29.5-33.9)$ & $30.0(22.6-33.8)$ & $14.9(13.0-16.7)$ & $26.5(22.3-30.8)$ & $8.9(5.8-12.1)$ \\
\hline \multicolumn{6}{|c|}{ Odds ratios for associations with prevalent $\mathrm{CHD}^{\mathrm{b}}(95 \% \mathrm{CI})$} \\
\hline \multicolumn{6}{|c|}{ WHO definition of the metabolic syndrome (all participants) } \\
\hline Number $^{\mathrm{c}}$ & 1,713 & 546 & 1,315 & 422 & 326 \\
\hline Unadjusted & $1.90(1.33-2.72)$ & $0.67(0.15-2.90)$ & $1.79(1.22-2.61)$ & $0.74(0.27-2.04)$ & $1.17(0.39-3.45)$ \\
\hline Adjusted for age & $1.64(1.14-2.37)$ & $0.53(0.12-2.31)$ & $1.60(1.09-2.36)$ & $0.67(0.24-1.86)$ & $1.27(0.41-3.90)$ \\
\hline $\begin{array}{l}\text { Adjusted for age and } \\
\text { smoking status }^{\mathrm{d}}\end{array}$ & $1.62(1.12-2.34)$ & $0.51(0.12-2.24)$ & $1.60(1.09-2.35)$ & $0.68(0.24-1.89)$ & $1.14(0.36-3.65)$ \\
\hline \multicolumn{6}{|c|}{ WHO definition of the metabolic syndrome (excluding those with known or newly diagnosed diabetes ${ }^{\mathrm{e}}$ ) } \\
\hline Number ${ }^{\mathrm{c}}$ & 1594 & 484 & 1031 & 284 & 262 \\
\hline Unadjusted & $1.46(0.94-2.26)$ & $\begin{array}{l}\text { All CHD cases were } \\
\text { without the meta- } \\
\text { bolic syndrome }\end{array}$ & $1.60(0.99-2.6)$ & $\begin{array}{l}\text { All CHD cases were } \\
\text { without the metabolic } \\
\text { syndrome }\end{array}$ & $1.40(0.37-5.29)$ \\
\hline Adjusted for age & $1.31(0.84-2.05)$ & & $1.48(0.91-2.40)$ & & $1.47(0.38-5.69)$ \\
\hline $\begin{array}{l}\text { Adjusted for age and } \\
\text { smoking status }\end{array}$ & $1.30(0.83-2.04)$ & & $1.50(0.92-2.44)$ & & $1.65(0.41-6.64)$ \\
\hline \multicolumn{6}{|c|}{ NCEP definition of the metabolic syndrome } \\
\hline Number ${ }^{\mathrm{c}}$ & 1,722 & 549 & 1,390 & 440 & 337 \\
\hline Unadjusted & $1.88(1.31-2.68)$ & $3.96(1.85-8.47)$ & $2.31(1.60-3.35)$ & $1.12(0.37-3.40)$ & $1.87(0.66-5.30)$ \\
\hline Adjusted for age & $1.65(1.15-2.38)$ & $3.26(1.50-7.09)$ & $2.04(1.40-2.97)$ & $1.05(0.34-3.21)$ & $2.02(0.69-5.92)$ \\
\hline $\begin{array}{l}\text { Adjusted for age and } \\
\text { smoking status }^{\mathrm{d}}\end{array}$ & $1.60(1.11-2.32)$ & $3.38(1.55-7.34)$ & $2.11(1.45-3.08)$ & $1.08(0.36-3.31)$ & $2.03(0.65-6.31)$ \\
\hline \multicolumn{6}{|c|}{ NCEP definition of the metabolic syndrome (excluding those with known or newly diagnosed diabetes ${ }^{\mathrm{e}}$ ) } \\
\hline Number ${ }^{\mathrm{c}}$ & 1,639 & 522 & 1,085 & 324 & 268 \\
\hline Unadjusted & $1.61(1.05-2.45)$ & $4.11(1.82-9.28)$ & $2.18(1.33-3.57)$ & $\begin{array}{l}\text { All CHD cases were } \\
\text { without the metabolic } \\
\text { syndrome }\end{array}$ & $3.57(1.11-11.5)$ \\
\hline Adjusted for age & $1.49(0.97-2.29)$ & $3.53(1.54-8.07)$ & $2.01(1.21-3.34)$ & & $3.76(1.15-12.33)$ \\
\hline $\begin{array}{l}\text { Adjusted for age and } \\
\text { smoking status }^{\mathrm{d}}\end{array}$ & $1.43(0.93-2.21)$ & $3.41(1.48-7.86)$ & $2.07(1.24-3.44)$ & & $3.81(1.09-13.21)$ \\
\hline
\end{tabular}

${ }^{\mathrm{a}}$ South Asian women were excluded due to the small number $(n=4)$ of CHD cases.

${ }^{\mathrm{b}} \mathrm{CHD}$ defined by doctor-diagnosed angina or heart attack, and/or the presence of major Q-waves or LBBB on ECG

${ }^{\mathrm{C}}$ Number available for analysis

${ }^{\mathrm{d}}$ Smoking status: current, ex, never

eAccording to WHO 1999 criteria (fasting plasma glucose $>7 \mathrm{mmol} / 1$ or OGTT plasma glucose $>11.1 \mathrm{mmol} / \mathrm{l}$ or known diabetes) [6] 
obesity criteria applied to $93 \%$ of South Asian men, whereas the more discriminatory waist circumference criteria provided by the NCEP applied to only $18 \%$ (Table 2 ).

Approximately $37 \%$ of participants were missing AER data. The majority of those with missing AERs had sufficient other data to assign metabolic syndrome status; 199 (4\%) participants could not be assigned WHO metabolic syndrome status due to missing AER data. Estimates of the prevalence of the metabolic syndrome (WHO definition) increased by only $0.06 \%$ when microalbuminuria was included in the definition.

Prevalence of $C H D$ The prevalence of CHD as defined by the presence of major ECG changes or a previous diagnosis was similar in European and South Asian men ( 9 and $10 \%$, respectively). European women and African-Caribbean men and women had lower prevalences of CHD (5-6\%). Only four (1.4\%) South Asian women had CHD, and these were excluded from analyses of associations between metabolic syndrome and CHD. Approximately twothirds of cases with prevalent CHD in all subgroups had been previously diagnosed by a doctor (Table 3 ).

Associations between the metabolic syndrome and CHD South Asian and European men with the NCEP definition of the metabolic syndrome were approximately twice as likely to have prevalent CHD as those without (Table 3). This association persisted after adjustment for age and smoking. Use of the WHO definition produced a similar association in European men, but a weaker association in South Asian men. In African-Caribbeans, no significant associations were apparent between the metabolic syndrome (either definition) and CHD, although the odds ratio (OR) for the NCEP definition was 2.03 (95\% CI 0.65 6.31) in women and the WHO definition appeared to confer some protection against $\mathrm{CHD}$ in men $(\mathrm{OR}=0.68,95 \% \mathrm{CI}$ $0.24-1.89$ ). In European women, there was a strong association between the NCEP definition of the metabolic syndrome and CHD (OR=3.4, 95\% CI 1.6-7.3), whereas this association was not present for the WHO definition of the metabolic syndrome $(\mathrm{OR}=0.51,95 \%$ CI $0.1-2.2)$.

The exclusion of participants with known or newly diagnosed diabetes (according to the criteria adopted by the WHO in 1999) reduced the associations between the two definitions of the metabolic syndrome and CHD in European and South Asian men. Conversely, these associations were increased in African-Caribbean women, with the most marked increase observed for the NCEP definition of the metabolic syndrome, where the adjusted OR was 3.8 $(p=0.04)$. In non-diabetic African-Caribbean men, all cases of CHD occurred in those without the metabolic syndrome (both definitions). In European women, the association between the NCEP definition of the metabolic syndrome and CHD was slightly higher in non-diabetic participants; however, there were no CHD cases in non-diabetic European women without the metabolic syndrome according to the WHO criteria (Table 3 ).
Comparison of the age-adjusted average values for the components of the metabolic syndrome for participants with and without prevalent CHD showed the expected trends of less favourable values in those with CHD. However, the differences between those with and without CHD were generally greater and more likely to be of statistical significance in Europeans than in other ethnic groups (see Table 1 of the Electronic Supplementary Material).

\section{Discussion}

This new analysis of the datasets from the Brent and Southall studies has identified marked differences in the prevalence of the metabolic syndrome between subgroups classified according to ethnicity and sex. South Asians had the highest overall prevalence of the metabolic syndrome, whereas Europeans had the lowest. Compared with the WHO definition, the NCEP definition provided lower prevalences of the metabolic syndrome in South Asian and African-Caribbean men, while the two definitions provided similar prevalences in South Asian and AfricanCaribbean women. Ethnic- and sex-specific variations in prevalence have also been observed in North American studies, but in different populations to those investigated in our study $[20,21]$.

Associations between the metabolic syndrome and prevalent CHD were strongest in European and South Asian men and, for the NCEP definition only, in European women; associations were weak or absent in African-Caribbean men - a pattern that was maintained when people with diabetes were excluded from the analyses. The exclusion of people with diabetes strengthened the association between the metabolic syndrome and CHD in African-Caribbean women. The observed associations between the metabolic syndrome and CHD in European men in this study are supported by findings from cross-sectional and prospective studies in European populations [7-22]. In our study, the metabolic syndrome as defined by the NCEP was more strongly associated with prevalent CHD than the WHO definition of the syndrome in all of the subgroups examined, apart from in European men. The San Antonio Heart Study found that the NCEP definition of the metabolic syndrome was more predictive of cardiovascular mortality than the WHO definition in lower-risk Mexican Americans and non-Hispanic white populations. Our findings of inconsistent or weak associations between prevalent CHD and the metabolic syndrome in European women and in African-Caribbeans may be related to the small numbers included in this study, but it is likely that absence of ethnic-group-specific validation of the metabolic syndrome definitions also contributes to the lack of association in African-Caribbeans. In general, compared with Europeans, UK African-Caribbean men tend to be insulin resistant and at high risk of diabetes, but have a favourable lipid profile and a low risk of CHD [23]. Due to this relative dissociation of insulin resistance and dyslipidaemia in African-Caribbeans, definition of the metabolic syndrome is problematic in this group. 
The central adiposity and impaired glucose homeostasis components contribute to the excess prevalence of the metabolic syndrome as defined by the WHO in South Asian and African-Caribbean men compared with that defined by the NCEP. The impaired glucose homeostasis component of the WHO definition of the metabolic syndrome includes insulin resistance, IFG, IGT and diabetes. However, the impaired glucose homeostasis component of the NCEP definition, intended for routine clinical use, requires only IFG and thus may omit people with glucose intolerance or diabetes whose fasting glucose is within normal limits. In contrast, the NCEP definition is weighted towards the inclusion of men and women with lesser degrees of dyslipidaemia or elevated blood pressure and of women with lower levels of obesity. Both of these tendencies may apply differentially to the different ethnic groups.

Within the WHO definition there is scope for variation in the estimation of insulin resistance. The formula used in this study is conceptually attractive in that it combines estimates of hepatic and peripheral insulin resistance and closely approximated the euglycaemic clamp in a study group of diabetic and non-diabetic patients. As noted by the European Group for the Study of Insulin Resistance (EGIR) [24], with regard to the upper quartile of insulin resistance, "it was not clear what the WHO consultation intended by the term "background population"...'. In this study we have used the entire non-diabetic study population as the background population as we believe this to be implied in the WHO definition. An alternative interpretation would involve the use of ethnic- and sex-specific upper quartiles, however, this automatically assigns $25 \%$ of all groups to the impaired glucose homeostasis category, and it is not clear that this approach is preferable given the known differences between ethnic groups in terms of cardiovascular risk.

The inclusion of microalbuminuria in the WHO definition has been challenged as unnecessary [15], while relevant data is seldom available in epidemiological studies. Extending the findings of the Data from an Epidemiological Study on the Insulin Resistance Syndrome (DESIR) study of European cohorts [24], we found that the presence of microalbuminuria had a minimal impact on the prevalence of the metabolic syndrome in any of the subgroups classified according to ethnicity and sex.

Both definitions of metabolic syndrome include widely accepted clusterings of risk factors for cardiovascular disease (CVD), and both are intended for clinical use as indicators of the risk of CVD. However, the variations in cutoff values, interpretations and the combination of components necessary to assign metabolic syndrome status result in substantial discordance between definitions. Importantly, neither definition recognises that the nature and contribution of each component may vary across ethnic groups. The metabolic syndrome definitions have been validated in European populations with reference to prediction of incident diabetes and CHD [7-13, 23], and modifications to the definitions have been suggested for use in European populations [24]. However, scant attention has been paid to the validation of the components and their cutoff values in other ethnic groups.
As in the US National Health and Nutrition Study [20], we found greater discordance between the WHO and NCEP definitions in non-Europeans.

In summary, this study demonstrates that, in a UK population, there are marked ethnic- and sex-specific variations in the prevalence of clusters of risk factors for CVD as described by recent definitions of the metabolic syndrome. In view of the observed variations in the association of the metabolic syndrome with prevalent CHD, a 'one size fits all' approach to defining the metabolic syndrome in different ethnic groups appears to be inappropriate. UK African-Caribbeans may be particularly poorly served by the current definitions of the metabolic syndrome given their differing risk factor profiles, and relatively low CHD mortality and high cerebrovascular mortality. In order for the metabolic syndrome to be an effective and practical indicator of cardiovascular risk, and hence a trigger for therapeutic intervention, prospective studies are needed to refine the definition by validating readily measurable components and their cutoff values against CVD morbidity and mortality in different ethnic groups.

Acknowledgements These analyses were supported by the British Heart Foundation. The Brent and Southall studies were supported by the UK Medical Research Council and the British Heart Foundation. I.F. Godsland is supported by the Heart Disease and Diabetes Research Trust.

\section{References}

1. Office for National Statistics (2002) Mortality statistics. Review of the Registrar General on deaths by cause, sex and age in England and Wales. The Stationery Office, London

2. Wild S, McKeigue PM (1997) Cross-sectional analysis of mortality by country of birth in England and Wales 1970-1992. BMJ 314:705-710

3. Reaven GM (1988) Banting lecture. Role of insulin resistance in human disease. Diabetes 37:1595-1607

4. McKeigue PM, Shah B, Marmot MG (1991) Relation of central obesity and insulin resistance with high diabetes prevalence and cardiovascular risk in South Asians. Lancet 337:382-386

5. Expert Panel on Detection, Evaluation, and Treatment of High Blood Cholesterol in Adults (2001) Executive summary of the third report of the National Cholesterol Education Program (NCEP) expert panel on detection, evaluation, and treatment of high blood cholesterol in adults (Adult Treatment Panel III). JAMA 285:2486-2497

6. World Health Organization, Department of Non-Communicable Disease Surveillance (1999) Definition, diagnosis and classification of diabetes mellitus. Part 1: diagnosis and classification of diabetes mellitus. World Health Organization, Geneva

7. Bonora E, Kiechl S, Willeit J et al (2003) Carotid atherosclerosis and coronary heart disease in the metabolic syndrome: prospective data from the Bruneck study. Diabetes Care 26:12511257

8. Lakka HM, Laaksonen DE, Lakka TA et al (2002) The metabolic syndrome and total cardiovascular disease mortality in middle-aged men. JAMA 288:2709-2716

9. Sattar N, Gaw A, Scherbakova O et al (2003) Metabolic syndrome with and without C-reactive protein as a predictor of coronary heart disease and diabetes in the West of Scotland Coronary Prevention Study. Circulation 108:414-419 
10. Ridker PM, Buring JE, Cook NR, Rifai N (2003) C-reactive protein, metabolic syndrome and risk of incident cardiovascular events: an 8-year follow-up of 14719 initially healthy American women. Circulation 107:391-397

11. Isomaa B, Almgren P, Tuomi T (2001) Cardiovascular morbidity and mortality associated with the metabolic syndrome. Diabetes Care 24:683-691

12. Hu G, Qiao Q, Tuomilehto J, Balkau B, Borch-Johnsen K, Pyorala K; DECODE Study Group (2004) Prevalence of the metabolic syndrome and its relation to all-cause and cardiovascular mortality in non-diabetic European men and women. Arch Intern Med 164:1066-1076

13. Hunt KJ, Resendez RG, Williams K, Haffner SM, Stern MP, San Antonio Heart Study (2004) National Cholesterol Education Program versus World Health Organization metabolic syndrome in relation to all-cause and cardiovascular mortality in the San Antonio Heart Study. Circulation 110:1251-1257

14. Chaturvedi N, McKeigue PM, Marmot MG (1993) Resting and ambulatory blood pressure differences in Afro-Caribbeans and Europeans. Hypertension 22:90-96

15. Balkau B, Charles MA (1999) Comment on the provisional report from the WHO consultation. European Group for the Study of Insulin Resistance (EGIR). Diabet Med 16:442-443

16. Matthews DR, Hosker JP, Rudenski AS, Naylor BA, Treacher DF, Turner RC (1985) Homeostasis model assessment: insulin resistance and beta-cell function from fasting plasma glucose and insulin concentrations in man. Diabetologia 7:412-419
17. Matsuda M, DeFronzo RA (1999) Insulin sensitivity indices obtained from oral glucose tolerance testing: comparison with the euglycemic insulin clamp. Diabetes Care 22:1462-1470

18. Godsland IF, Jeffs JAR, Johnston DG (2004) Loss of beta cell function as fasting glucose increases in the non-diabetic range. Diabetologia 47:1157-1166

19. Mattix HJ, Hsu CY, Shaykevich S, Curhan G (2002) Use of the albumin/creatinine ratio to detect microalbuminuria: implications of sex and race. J Am Soc Nephrol 13:1034-1039

20. Ford ES, Giles WH (2003) A comparison of the prevalence of the metabolic syndrome using two proposed definitions. Diabetes Care 26: 575-581

21. Anand SS, Yi Q, Gerstein H et al (2003) Relationship of metabolic syndrome and fibrinolytic dysfunction to cardiovascular disease. Circulation 108:420-425

22. Laaksonen DE, Lakka HM, Niskanen LK, Kaplan GA, Salonen JT, Lakka TA (2002) Metabolic syndrome and development of diabetes mellitus: application and validation of recently suggested definitions of the metabolic syndrome in a prospective cohort study. Am J Epidemiol 156:1070-1077

23. Chaturvedi N, McKeigue PM, Marmot MG (1994) Relationship of glucose intolerance to coronary risk in Afro-Caribbeans compared with Europeans. Diabetologia 37:765-772

24. The European Group for the Study of Insulin Resistance (2002) Frequency of the WHO metabolic syndrome in European cohorts, and an alternative definition of an insulin resistance syndrome. Diabetes Metab 28:364-376 